\title{
Analysis of Information and Communications Technology (ICT) for the Internet-based business
}

\author{
B. G. Lee \& Y. H. Lee \\ Graduate School of Information, Yonsei University, Seoul, Korea
}

\begin{abstract}
This study analyzes the trends of ICT (Information and Communications Technology) with value chains of the Internet-based business. We build a technical roadmap of ICT for investigating its content, network and platform. In this paper we focus on the gradual transition of technology and summarize the trends of ICT as three categories. Even though the existing value chains of the ICT business and industry still endure, the innovative value chains are emerging vertically as well as horizontally not only within the ICT industry but also between other industries. The advances of ICT can create enormous business opportunities. However, in Internet-based business and industry, there are still obstacles and barriers to overcome. Thereby, Internet-based business enterprises will require adoption of the ICT convergence business strategy and/or the divergence strategy. The results of the study can serve as useful guidelines for similar industries as well as other countries.

Keywords: Internet-based business, ICT (Information and Communications Technology), technical roadmap, digital convergence, value chain.
\end{abstract}

\section{Introduction}

The advances of ICT (Information and Communications Technology) and convergence technologies have generated diverse digital products as well as increasing demands for the Internet-based business. The digital convergence of ICT can be regarded as the unification of functions: the coming together of previously distinct products that employ digital technology [1]. For example, the PDA, which is composed of the telephone function and the computer, is a typical digital convergence product. Both utilize digital technologies, but they 
historically served completely different markets with totally different functions. That is, as shown in Figure 1, the environments for the Internet-based business have been changed entirely. Thereby even though many convergence products have been produced, it was not easy for them to success in the markets. Numerous companies have sought, and failed, to take digital convergence with a big technological breakthrough [2].

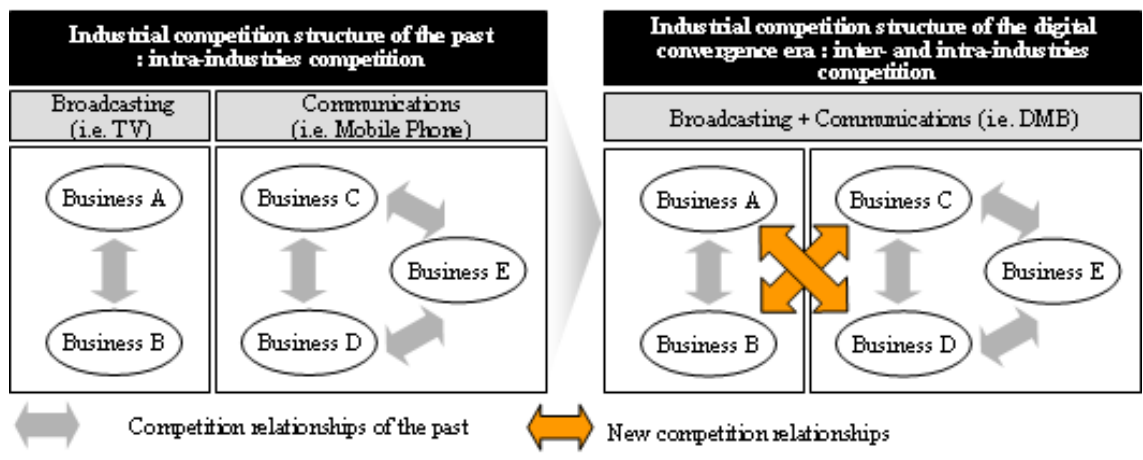

Figure 1: Industrial structure and competition.

How can we understand the consequences of digital convergence in the Internet-based business environments? Until recently, there are very few studies regarding the relationships between the trends of ICT and the Internet-based business $[3,4]$. The argument in this article is that the technical roadmap of ICT presents not only the development of complete convergence but also several types of value chains for the Internet-based business.

We in this study build a technical roadmap and examine some problems with being serve digital convergence. The technical roadmap is a useful tool to explore trends of ICT contents and products and it is one of the most widely used methods for supporting the strategic management of technology. It also helps to forecast technological future trends based on either exploratory methods or normative approaches at the industry level and provides a graphical means exploring and communicating the relationship among markets, products, and technologies over time $[5,6]$.

We in this paper focus on the gradual transition of technology and summarize the trends of ICT as three categories: 'One for One', 'One for All' (and/or 'All for One') and 'All for All'. The abbreviation of 'One for One' represents that one content makes for one user platform. 'All for All' denotes that diverse contents operate in various user platforms via multiple networks.

We develop and analyze the value chain of the Internet-based business using the technical roadmap. For building the roadmap, secondary data from public and private research centers are utilized. The data have been collected from 1950 to 2012 and reconstructed by the industrial structure and value chain standards. To make and investigate value chain models, the scope of ICT in this 
study is restricted. For example, this paper represents TV as broadcasting and the telephone as communications.

\section{One content for one platform}

Before the appearance of digital convergence technology, broadcasting and communications sustained their own technologically and legally distinct territories, and telephones and TV developed and evolved within those respective territories [2]. In that era, the launch of a new phone with new-fangled functions didn't mean anything more than telephonic communication, an exchange of voices. In the side of communications, even though TV developed by adding color and better resolution, it is imposed by the broadcasting industry just by providing a one-way service [7].

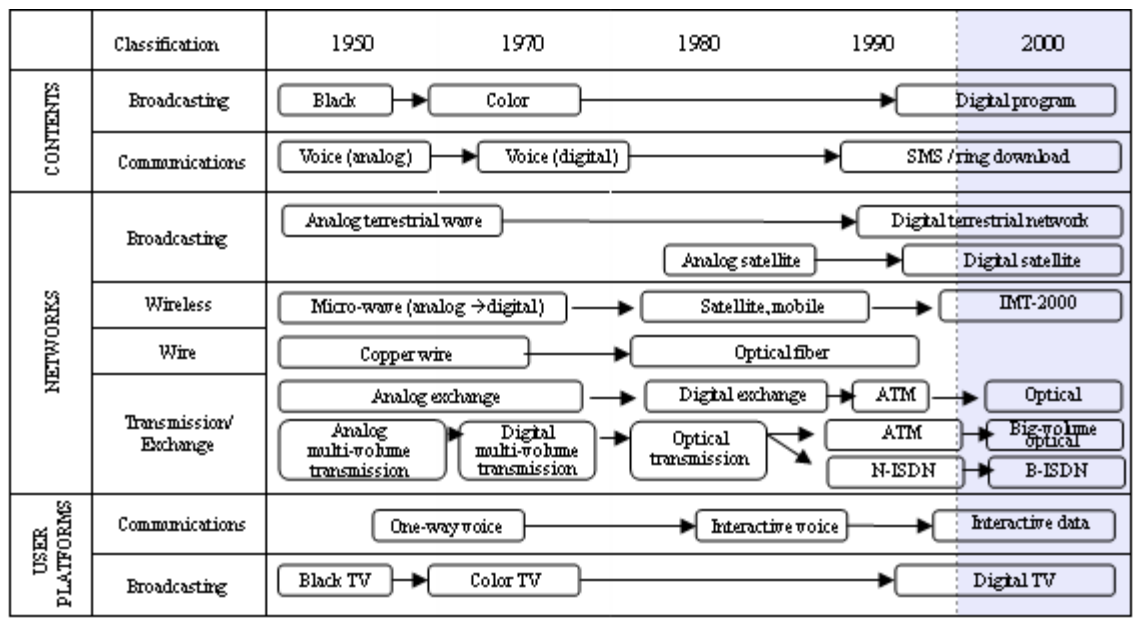

Figure 2: $\quad$ Technical roadmap in the stage of 'One for One'.

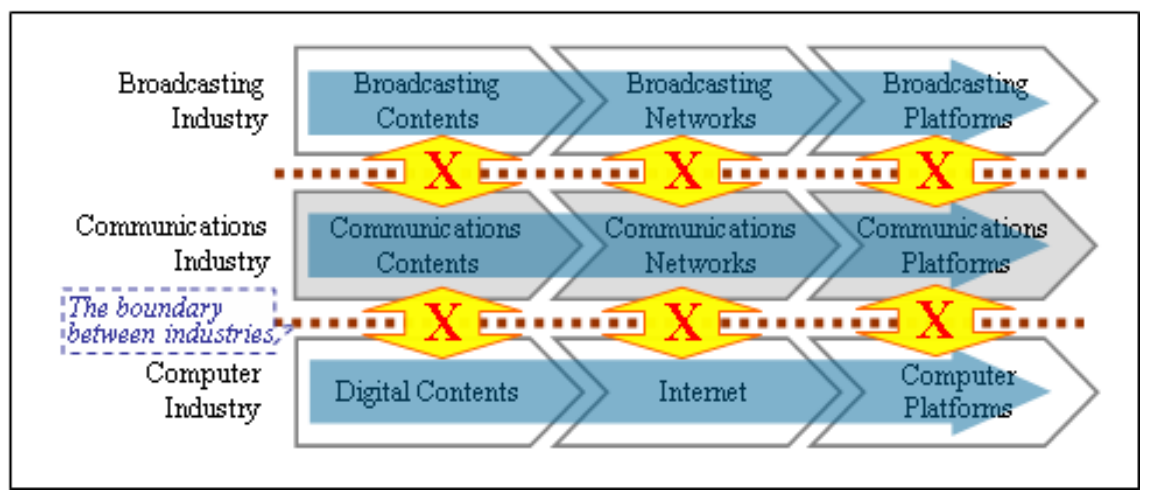

Figure 3: Industrial structure and value chain in the stage of the 'One for One'. 
When a new model of TV or telephone reached the market, its business was strictly limited to a 'One for One' service structure. The 'One for One' service means that one content makes for one user platform. In this stage, it seems that many companies didn't consider various synergy effects of digital convergence although they have the digital technology. Figure 2 illustrates the trends of ICT in industries of broadcasting and communications [8].

Figure 3 depicts the value chains of industries of broadcasting and communications with the industrial structures and boundary relationships. Each industry has its own business boundary with the value chain and the boundary between industries is clearly divided into each other.

In this stage, the Internet-based business enterprises including Amazon, eBay and Yahoo had established and grown through wired networks. However, there had been various limitations and obstacles to overcome such as problems of content, device, security and wireless networks.

\section{One content for various platforms and/or diverse contents for one platform}

The businesses of broadcasting and communications have recently become interactive and made a smooth convergence [9]. For example, we can send a digital image via telephone lines to any number of people. And unlike in the past, broadcasting contents can be sent not only to TV monitors, but also to different devices such as mobile phones and PCs. These are good examples of 'One for All/All for One' services. 'One for All' and/or 'All for One' means that one content operates on various platforms and/or diverse contents work in one platform $[10,11,12,13]$.

From this stage, the innovative contents, networks and devices for the Internetbased business are emerging and becoming more sophisticated.

\subsection{Contents technology}

Contents in broadcasting and communications are changing into digital forms based on the multi-media converging systems to promote user-friendliness, dynamic expression, or responsiveness. In order to provide numerous contents through various networks, technical improvements in ICT such as image processing and transmitting have been implemented.

As contents converged to digital, the distinction between contents such as sound and data disappeared. New up-and-coming Internet applications including Telematics services are emerging. Thereby, the size and variety of contents have increased radically.

\subsection{Network technology}

As networks converge and develop to digital, the usability of network can be increasingly optimized. Advances in digital convergence technologies accelerate the integration and hybridization of different networks. Also, the government 
has promoted the nationwide network infrastructures such as $\mathrm{BcN}$ (Broadband Convergence Network) and USN (Ubiquitous Sensor Network). New wired and wireless network technologies and services including DMB (Digital Multimedia Broadcasting) and PLC (Power Line Communications) are emerging. The demands for the extra communications bandwidth and the standardization are increasing.

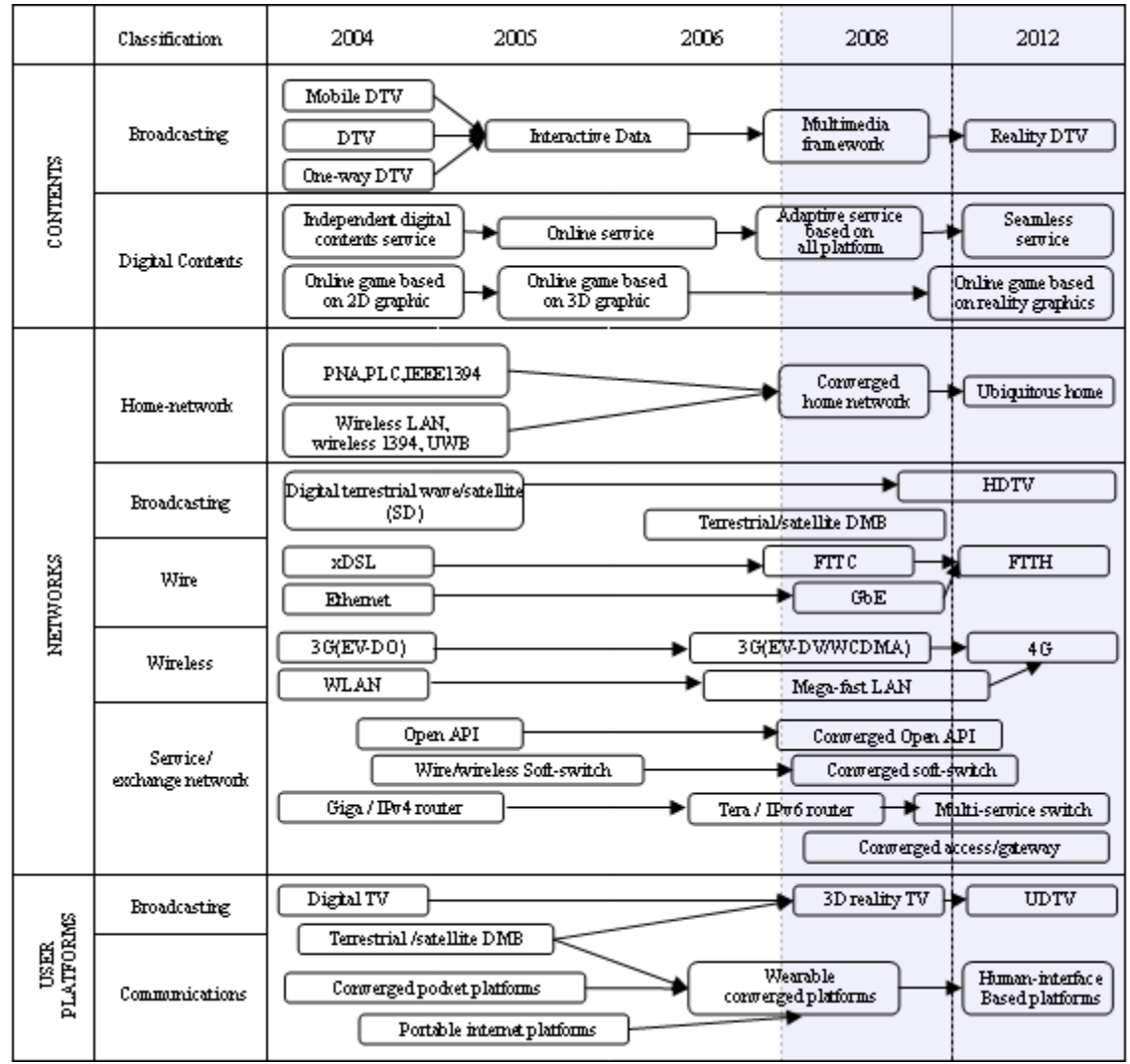

Figure 4: Technical roadmap for 'One for All and/or All for One' and 'All for All'

Figure 4 addresses the main features of network technologies including IPv6 (Internet Protocol version 6), FTTH (Fiber to the Home) and 3G/4G (generation). $3 \mathrm{G} / 4 \mathrm{G}$ technologies accelerate the integration of wired and wireless service. IPv6 guarantees the user's mobility by integrating wired and wireless service; it is a key technology for using Internet and Post PC service on mobile phones.

In this stage, the network embodies many new technologies and enables optical transmission and the optimization of valuable services such as IPTV (Internet Protocol Television) [14]. 


\subsection{Platform technology}

Advanced convergence technology enables a user platform to have various functions such as cellular phone, MP3 player, PDA and digital camera. For example, the terrestrial DMB phone has the functions of broadcasting and communications in one device. The technology of DMB lies in connecting the two services, so that the user can freely move between broadcasting and communication services without converting it to a physical transfer network or platform [15]. The device of IPTV is another good example, i.e., it presents the Internet service and TV on one screen simultaneously.

'All for One' service structure enables users to access all types of content. The more convergence technologies mature, the more user platforms with multiple functions progress. Sometimes the functional complexities of user platform can cause to have not only inconvenience but redundant costs. As the success case of niche market, a simple low-priced cellular phone can be a good example. In summary, the new value chains within the ICT business and industry are coming out vertically as well as horizontally.

\section{Diverse contents for various platforms}

The shaded areas in Figure 4 represents the stage of 'All for All' that denotes 'diverse contents operate on various user devices via multiple networks.' The advances of ICT in the near future can support more complete convergence services on almost the whole range of the Internet-based business. Convergence products and services have emerged and extended to various businesses. Fortunately, the applied scope of ICT extends more and more, as shown in Figure 5. The ICT with digital convergence can create great business opportunities especially for the Internet-based business. The more services converge, the more business opportunities occur. After all, the killer applications of ICT in the Internet-based business are increasing radically.

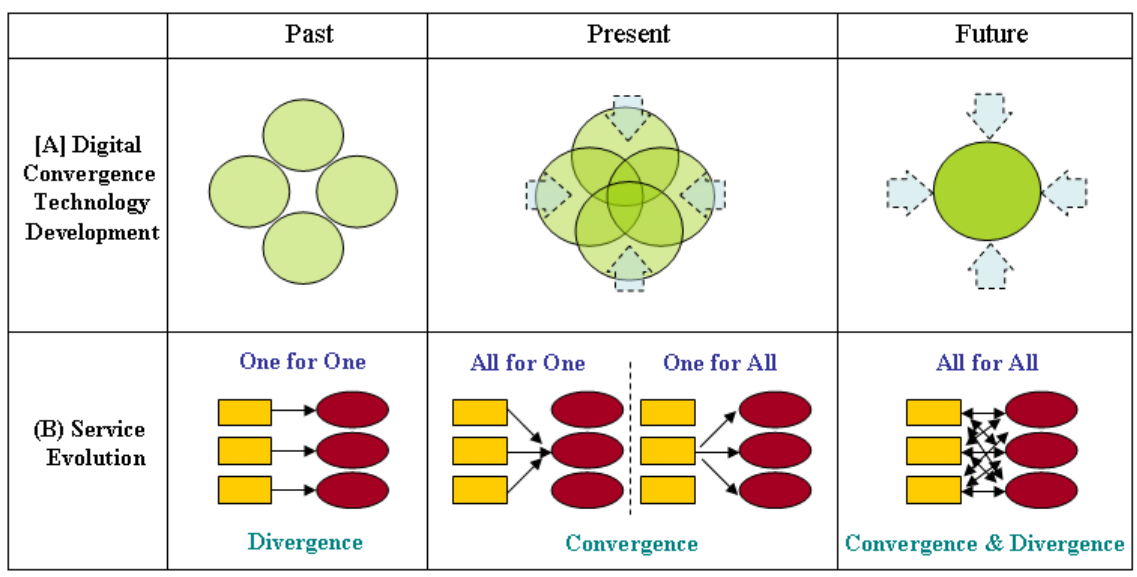

Figure 5: The digital convergence service type 
However, in the Internet-based business and industry, there are still obstacles and barriers to overcome. Since overqualified contents, networks, platforms, and products can bring about some dissipation like the expensive ICT device that has unnecessary functions. The Internet-based business enterprises should consider the ICT convergence business strategy and the divergence strategy simultaneously.

Anyway, in this stage, the existing value chain of the ICT industry still endures as shown in the bottom illustration of Figure 6. Furthermore, the innovative value chains not only within the ICT industry but also between other industries will settle down vertically as well as horizontally.

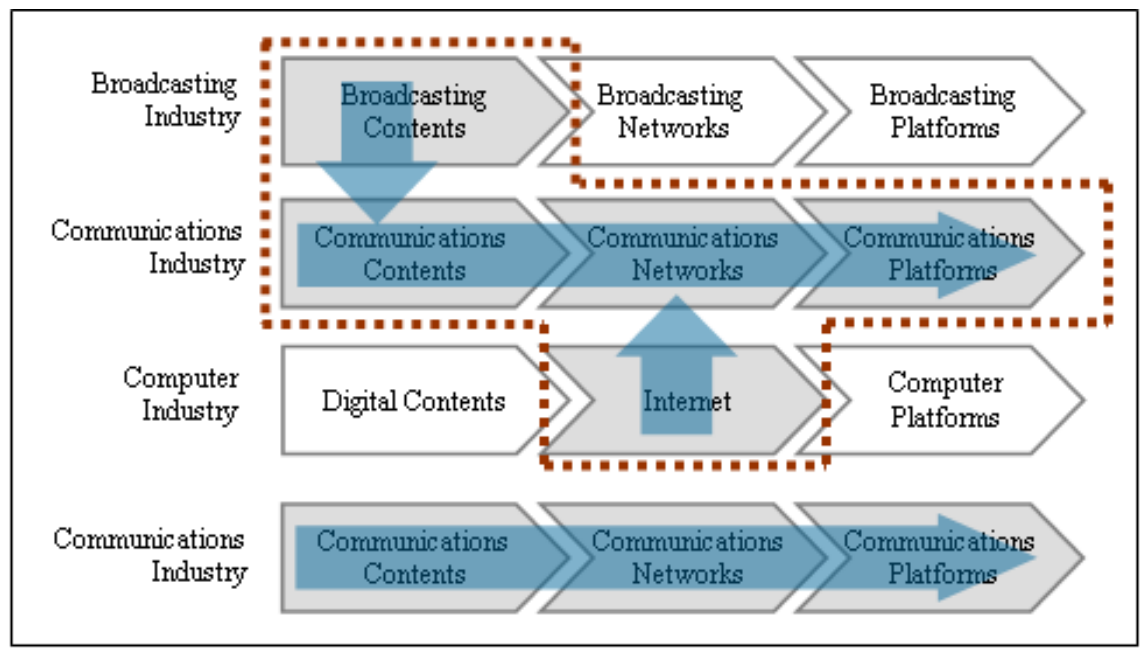

Figure 6: Industrial structure and value chain in the stage of 'All for All'.

\section{Conclusion}

The great advantage of ICT is its digital convergence capability. The diffusion of ICT is not limited to contents. It can be applied to networks, platforms, information transfer and exchanging technologies. Furthermore, it leads to innovative environments for the Internet-based business including products, services and industries.

The trends of ICT based on the technical roadmap can be classified three groups in terms of 'One for One', 'All for One (and/or One for All)', and 'All for All'. Technical movements have shifted from 'One for One' to 'All for One (and/or One for All)', finally to 'All for All'.

In the stage of 'One for One', one content in the ICT industries makes for one user platform, mainly because of technical restrictions. Each industry has its own business boundary with the value chain and the boundary between industries is clearly divided into each other. The Internet-based business enterprises had established and grown via wired networks. 
In the period of 'All for One (and/or One for All)', one content operates on various user devices and/or diverse contents work in one platform. The boundaries of business and industry become collapsing. And the value chain factors such as contents, networks, platforms, and products have partially become interactive and made convergence. From this stage, the innovative contents, networks and devices for the Internet-based business are emerging and becoming more sophisticated.

In the era of 'All for All', all factors relating to the value chain of the Internetbased business can be converged. The ICT with digital convergence can generate enormous business opportunities. However, in the Internet-based business and industry, there are still obstacles and barriers to overcome. Overqualified contents, networks, platforms, and products can bring about preventable dissipation such as the expensive ICT device with unnecessary functions. The Internet-based business enterprises will require adoption of the ICT convergence business strategy and/or the divergence strategy.

The results of this study can serve as useful guidelines for similar industries as well as other countries. However, results from this study have several limitations to overcome for analyzing feasibly emerging technologies, human behaviors and societies. Particularly, in the further research, the study needs more interdisciplinary approaches because the success of the Internet-based business is depended upon lots of significant factors such as Law and legal issues, government policy, standardization, QoS (Quality of Service), security, etc.

\section{Reference}

[1] Lee, Y. H., Song, J. Y., Kim, S. G. \& Lee, B. G., Beyond the digital convergence. Proceedings of the $4^{\text {th }}$ International Conference on Asian Language Processing and Information Technology, pp. 269-276, 2005.

[2] Yoffie, D. B., Competing in the age of digital convergence. California Management Review, 38(4), pp. 31-53, 1996.

[3] Kothandaraman, P. \& Wilson, D. T., The future of competition: valuecreating networks. Industrial Marketing and Management, 37, pp. 379389, 2001.

[4] Maitland, C. F., Bauer, J. M. \& Westerveld, R., The European market for mobile data: evolving value chains and industry structures. Telecommunications Policy, 26, pp. 485-504, 2002.

[5] Lee, S. J. \& Park, Y. T., Customization of technology roadmaps according to roadmapping purposes: overall process and detailed modules. Technological Forecasting \& Change, 72, pp. 567-583, 2005.

[6] Kappel, T. A., Perspectives on roadmap: how organizations talk about the future. Product Innovation Management, 18(1), pp. 39-50, 2001.

[7] Kim, D. H., The prospect and trend in digital TV market, 2004, Korea Electronics Technology Institute, Seoul, South Korea.

[8] Korea Telecom (KT), Information telecommunication history, Seoul, http://museum.kt.co.kr 
[9] Ryu, J. C. et al., Digital Convergence, Communication Books Publication: Seoul, pp. 52-434, 2004.

[10] Lee, T. W., Multimedia technology roadmap, 2002, Korea Industrial Technology Foundation, Seoul, South Korea.

[11] Choi, Y. S., Digital TV technology roadmap. Electronic Information Center, pp. 1-13, 2004.

[12] Ha, S. W., Mobile telecommunication technology roadmap. Electronic Information Center, pp. 1-11, 2004.

[13] Electronic Times, The almanac of information telecommunication, 2005, Electronic Times, Seoul, South Korea.

[14] Telecommunication Technology Association (TTA), Information communication standardization, 2002, TTA, Seoul, South Korea.

[15] Lim, Y. K., Multimedia broadcasting technology. TTA Journal, 98, pp.115-121, 2005. 